\title{
ANÁLISE DA MORTALIDADE MATERNA DO MUNICÍPIO DE RIBEIRÃO PRETO-SP - NO PERÍODO DE 1991 A $1995^{1}$
}

Oba MDV, Tavares MSG. Análise da mortalidade materna do município de Ribeirão Preto-SP - no período de 1991 a 1995. Rev Latino-am Enfermagem 2001 maio; 9(3):70-6.

O objetivo deste estudo foi conhecer e identificar as causas de morte materna. Os dados foram obtidos junto ao Comitê de Estudos e Prevenção de Morte Materna de Ribeirão Preto e atestados de óbitos. É um estudo exploratório, onde foram analisados 72 óbitos maternos, ocorridos em Ribeirão Preto, durante o período de 1991 a 1995. Em 1995 o coeficiente de mortalidade materna foi de 60,3 por 100.000 nascidos vivos, com predomínio de causas evitáveis, como a hemorragia (33,3\%), outras causas (26,4\%), hipertensão (15,3\%), aborto (11,1\%), infecção (8,3\%) e puerpério (5,6\%). A mortalidade materna é um problema de saúde pública, que deve ser enfrentado pelas autoridades governamentais.

UNITERMOS: mortalidade, mortalidade materna, saúde da mulher

\section{ANALYSIS OF MATERNAL MORTALITY IN THE MUNICIPALITY OF RIBEIRÃO PRETO-SP FROM 1991 TO 1995}

This study aims at learning about and identifying the causes leading to maternal death. Data were obtained from the Ribeirão Preto Committee for Studies and Prevention of Maternal Death and from obituaries. It is an exploratory study in which 72 maternal deaths occurring in Ribeirão Preto were analyzed from 1991 to 1995. In 1995, the maternal death rate was 60.3 in 100,000 births, with the predominance of avoidable causes, such as hemorrhage (33.3\%), other causes (26.4\%), hypertension (15.3\%), abortion (11.1\%), infection (8.3\%) and puerperium (5.6\%). Maternal death is a public health problem which must be faced by government authorities.

KEY WORDS: mortality, maternal mortality, women's health

\section{ANÁLISIS DE LA MORTALIDAD MATERNA DEL MUNICIPIO DE RIBEIRÃO PRETO-SP - EN EL PERÍODO DE 1991 A 1995}

El objetivo de este estudio es conocer e identificar las causas de muerte materna, los datos fueron obtenidos en el Comité de Estudio y Prevención de Muerte Materna de Ribeirão Preto y certificados de defunciones. Es un estudio exploratorio, en el que fueron analizados 72 defunciones maternas ocurridas en Ribeirão Preto, durante el período de 1991 a 1995. En 1995 el coeficiente de mortalidad materna es de 60,3 en cada 100.000 nacidos vivos en 1995, con predominio de causas evitables, como la hemorragia (33,3\%), otras causas (26,4\%), hipertensión (15,3\%), aborto (11,1\%), infección (8,3\%) y puerperio (5,6\%). La mortalidad materna es un problema de salud pública que debe ser enfrentado por las autoridades gubernamentales.

TÉRMINOS CLAVES: mortalidad, mortalidad materna, salud de la mujer

\footnotetext{
${ }^{1}$ Pesquisa apresentada no VI Colóquio Pan-Americano de Investigação em Enfermagem, organizado pela Escola de Enfermagem de Ribeirão Preto da Universidade de São Paulo em 19 a 22/5/1998

2Doutor em Enfermagem, Enfermeira da Secretaria Municipal da Saúde de Ribeirão Preto. E-mail: mariadasdores@uol.com.br

${ }^{3}$ Professor Doutor em Enfermagem e orientadora do Programa de Doutoramento em Enfermagem do Programa Interunidades da Escola de Enfermagem e Escola de Enfermagem de Ribeirão Preto da Universidade de São Paulo, Centro Colaborador da OMS para o desenvolvimento da pesquisa em Enfermagem
} 


\section{INTRODUÇÃO}

A mortalidade materna é um dos mais fiéis e importantes indicadores de saúde de um povo. Estima-se que a cada ano ocorram no mundo meio milhões de mortes maternas, sendo que mais de 99\% delas ocorrem nos países do terceiro mundo. Na América Latina morrem anualmente em torno de 30000 mulheres por complicações da gravidez, parto e puerpério ${ }^{(1)}$.

A morte de mulheres durante a gestação e/ou puerpério por causas direta ou indiretamente ligadas á gravidez (morte materna) representa enorme parcela de óbitos em adultos em todo mundo, especialmente nos países subdesenvolvidos da África, América Latina e, neste principalmente o $\mathrm{Brasil}^{(2)}$.

0 sistema de saúde implantado no Brasil tem mostrado um quadro extremamente desanimador, caracterizado pela ineficácia e/ou ineficiência de atendimento, inadequado às necessidades da população e com insuficiência de recursos materiais, humanos e financeiros. A assistência está concentrada nos hospitais em detrimento do serviço preventivo de saúde ${ }^{(3)}$.

Pode-se dizer que a assistência pré-natal é um fator importante na redução da mortalidade materna e perinatal, visto que, muitas patologias no período gravídico-puerperal podem ser tratadas e/ou controladas, evitando efeitos danosos. É preciso lembrar também que um pré-natal bem feito, certamente, orientará no sentido de se evitar problemas específicos do parto ou mesmo cuidados imediatos do recém-nascido, além daqueles do período puerperal.

Observa-se que a reorganização da assistência à saúde da mulher após a municipalização em Ribeirão Preto sofreu modificações em decorrência da dinâmica da política de saúde vigente no município, ou seja, centralizadora, um modelo assistencial que é de pronto atendimento e que tem ação central à consulta médica, com reconhecimento individual dos problemas e a solução dada pela prática médica, essencialmente curativa ${ }^{(4)}$.

0 motivo da procura das gestantes a estas Unidades Básicas Distritais de Saúde refletiu a necessidade de manutenção de sua saúde e do concepto, assim como, a possibilidade de utilizar toda a tecnologia disponível, para este tipo de assistência pré-natal (ultra-sonografia, cardiotocografia, laboratório de análise clínica etc.). As Mulheres buscam assistência na rede pública de saúde responsável em prestar assistência de qualidade e integral, não só no que diz respeito ao ciclo reprodutivo, mas a todo ciclo vital ${ }^{(5)}$.

Entretanto estas mulheres deparam-se com um atendimento, que consolida a assistência médica como prática hegemônica, onde as consultas são ligadas basicamente às queixas e condutas, na realização dos exames físicos e tocoginecológicos e à solicitação de exames complementares, não havendo tempo nem espaço para fala e/ou questionamento destas mulheres. 0 atendimento é mais ritualístico do que preventivo ou resolutivo ${ }^{(6)}$.

Pesquisas envolvendo a morte materna devem ser estimuladas principalmente em países como o Brasil que apresenta índices de mortalidade absurdamente altos, mesmo quando comparado com países menos desenvolvidos economicamente. Certamente o primeiro passo para que se possa desenvolver uma política adequada de combate à mortalidade materna é o conhecimento correto de suas causas e de sua magnitude ${ }^{(7)}$.

0 coeficiente de mortalidade materna(CMM) é internacionalmente reconhecido como parâmetro confiável, entre outros índices de avaliação da saúde pública, porém inexplicavelmente é quase desconhecido entre nós.

As elevadas cifras de óbitos maternos são decorrentes das causas obstétricas diretas em nosso meio, o que torna imprescindível o estudo das características destes óbitos, a fim de que sejam adotadas medidas que possam reduzir a sua incidência ${ }^{(8)}$.

O OBJETIVO deste estudo é conhecer e identificar as causas que levaram a morte materna, tendo como base a investigação dos óbitos maternos ocorridos em Ribeirão Preto, através dos dados obtidos junto ao Comitê de Estudo e Prevenção de Morte Materna e Declarações de Óbitos.

\section{CARACTERIZAÇÃO DA ÁREA EM ESTUDO}

Este trabalho é um estudo exploratório, onde foram analisados 72 declarações de óbitos maternos ocorridos em Ribeirão Preto, no período de 1991 a 1995.

A população geral do município de Ribeirão Preto, com base nos dados da Fundação Sistema Estadual de Análise de Dados (SEADE) de 1995, é de 450.995 habitantes, sendo que $99 \%$ residem na zona urbana. 0 sexo feminino é representado por 232576 habitantes (52\%).

Verifica-se que, no ano de 1995, ocorrem 11431 nascimentos, com um coeficiente de natalidade geral de 18,62\%. 0 maior número de nascimentos ocorreu no setor norte do município 3758 , seguido pela região oeste com 2681 nascimentos, conforme dados obtidos na Divisão de Informática da Secretaria Municipal da Saúde de Ribeirão Preto. De acordo com o censo do IBGE de 1991, o setor norte da cidade é o que concentra maior população 179 849, seguida da região oeste com 135509 habitantes.

De acordo com os dados obtidos na Divisão de Informática da Secretaria Municipal da Saúde de Ribeirão Preto, a cidade está dividida em cinco Distritos de Saúde; a rede municipal é composta por cinco Unidades Básicas Distritais de Saúde (UBDS) e vinte duas Unidades Básicas de Saúde (UBS), três núcleos de assistência à saúde mental e um ambulatório regional de especialidades (NGA59). 
No ano de 1995 foram realizadas 96106 consultas na especialidade de ginecologia e obstetrícia. Não foi possível identificar quantas consultas eram de pré-natal. Foram realizados 11.431 partos, sendo $54 \%$ cirúrgicos (cesária), $43 \%$ normais e $2 \%$ fórceps e $1 \%$ ignorado, segundo dados obtidos junto a Divisão de Informática da Secretaria Municipal da Saúde de Ribeirão Preto.

\section{RESULTADOS}

Os resultados da análise dos 72 óbitos maternos, ocorridos em Ribeirão Preto no período de 1991 a 1995, estão apresentados nos gráficos e tabelas abaixo descritos.

Os números anuais de óbitos oscilam entre nove a dezoito, sendo que as mulheres, residentes na cidade de Ribeirão Preto, representaram $27 \%$ a $47 \%$ dos óbitos maternos no período de 1991 a 1995, conforme observa-se na Tabela 1.

Tabela 1 - Morte materna ocorrida em Ribeirão Preto, de acordo com a localidade, no período de 1991 a 1995*

\begin{tabular}{|c|c|c|c|c|c|c|c|c|c|c|c|c|}
\hline & 19 & 91 & & 992 & 19 & 93 & 199 & 194 & & 995 & & otal \\
\hline Localidade & $N$ & of & $\mathrm{N}$ & of & $N$ & of & $\mathrm{N}$ & of & $\mathrm{N}$ & of & $N$ & $\%$ \\
\hline Ribeirão Preto & 4 & 27 & 7 & 47 & 4 & 44 & 7 & 47 & 5 & 28 & 27 & 37 \\
\hline $\begin{array}{l}\text { Outros municípios } \\
\text { da DIRXYill }\end{array}$ & 7 & 46 & 5 & 33 & 2 & 23 & 7 & 47 & 6 & 33 & 27 & 3 \\
\hline Outras localidades & 4 & 27 & 3 & 20 & 3 & 33 & 1 & 6 & 7 & 39 & 18 & 25 \\
\hline Total & 15 & 100 & 15 & 100 & 9 & 100 & 15 & 100 & 18 & 100 & 72 & 100 \\
\hline
\end{tabular}

Observa-se preenchimento inadequado das declarações dos óbitos maternos, mesmo nos campos das declaraç̃̃es, em que o profissional médico só tenha necessidade de assinalar. Em conseqüência deste fato, não foi possível obter dados referentes ao número de filhos, duração da gestação, etc.

Constata-se que o grau de instrução predominante é 0 fundamental, ou seja, 44,4\%. Observa-se também que, em 55,6\% das declarações, não consta esta informação. A ocupação predominante destas mulheres é do lar em $73,6 \%$; apenas $26,4 \%$ destas trabalham fora de casa, sendo $50 \%$ trabalham como domésticas. Com relação ao estado civil das mulheres que foram à óbito observa-se predomínio de casadas (54\%), solteiras (32\%), viúvas (3\%), desquitadas (1\%), ignorados (4\%) e não consta (6\%).

As dificuldades com relação aos dados de mortalidade materna começam na interpretação dos atestados de óbitos. Percebe-se uma tendência ao descuido no preenchimento destes certificados, que muitas vezes são tratados como mera ocorrência formal. A negligência dos que os assinam falseia os resultados oficiais $^{(9)}$.
Uma dificuldade apontada para desenvolvimento do trabalho do Comitê de Morte Materna foi a piora na qualidade do preenchimento das declarações. Cita ainda que não se pode descartar a possibilidade de uma piora gradativa na qualidade dos profissionais envolvidos no preenchimento da declaração de óbito ${ }^{(10)}$.

As mortes maternas representam a $8^{\text {a }}$ causa de óbitos em mulheres de 10 a 49 anos, conforme 0 artigo "A absurda mortalidade materna", publicado em Medicina Conselho Federal ${ }^{(11)}$. Os óbitos maternos ocorridos em Ribeirão Preto, observa-se que estes ocorreu na faixa de 13 a 45 anos, com predomínio na faixa etária de 20 a 34 anos (67\%). 0 dado obtido na pesquisa é semelhante ao obtido na investigação da mortalidade materna no município de São Paulo ${ }^{(12)}$, que relata concentração de óbitos maternos na faixa etária entre 20 e 34anos (67,5\%). No estudo realizado na Maternidade Dona Evangelina Rosa ${ }^{(8)}$ foi de 20 a 30 anos $(57,14 \%)$, conforme Figura 1.

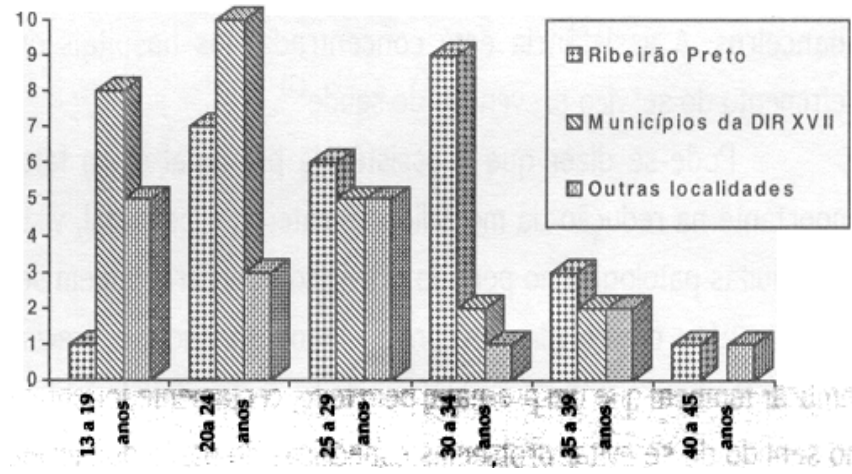

* Fonte: Secretaria Municipal da Saúde de Ribeirão Preto-SP e Comitê de Estudo e Prevenção de Morte Materna

Figura 1 - Morte materna ocorrida em Ribeirão Preto de acordo com a faixa etária e localidade no período de 1991 a 1995*

A mortalidade materna, conjuntamente com a mortalidade perinatal, traduz a eficácia dos serviços de saúde, avaliando o acesso ao sistema, a freqüência ao pré-natal e a assistência ao parto e puerpério. Esperar-se-ia que, quanto melhor equipado e estruturado o serviço de saúde de um determinado local, menor seriam seus índices de mortalidade materna ${ }^{(13)}$.

Ao invés disso, o Brasil possui uma situação paradoxal, isto é, hospitais de nível terciário, a exemplo do Hospital São Paulo (Universidade Federal de São Paulo), apresentam elevados coeficientes de mortalidade materna, em razão de serem centros de referência em um sistema de péssima qualidade, destroçado pela crônica falta de recursos e de planejamento, onde não existe adequada distribuição no atendimento primário e secundário ${ }^{(13)}$.

Além disso, observa-se a falta de um sistema hierarquizado de saúde, fazendo com que os centros de atendimentos primários não tenham outra alternativa, a não ser encaminhar os casos mais graves para hospitais que teoricamente teriam melhor capacidade de resolução ${ }^{(13)}$. 
Na investigação da mortalidade materna no município de São Paulo verifica-se que a maioria dos óbitos maternas ocorrem dentro do ambiente hospitalar. Salienta-se que o maior número de partos, na capital paulista ocorrem na rede privada/conveniada ${ }^{(12)}$.

Nos hospitais universitários onde teoricamente havia melhor capacidade de resolução, observa-se um retardamento adicional e a utilização de esquemas terapêuticos, muitas vezes absurdos, além da subestimação de sinais e sintomas clínicos, claramente relacionados com situações de emergência, contribuindo desta forma para ocorrência de morte materna ${ }^{(13)}$.

Em Ribeirão Preto, o Hospital das Clinicas da Faculdade de Medicina da Universidade de São Paulo, apresenta a maior concentração de mortes maternas, conforme Figura 2.

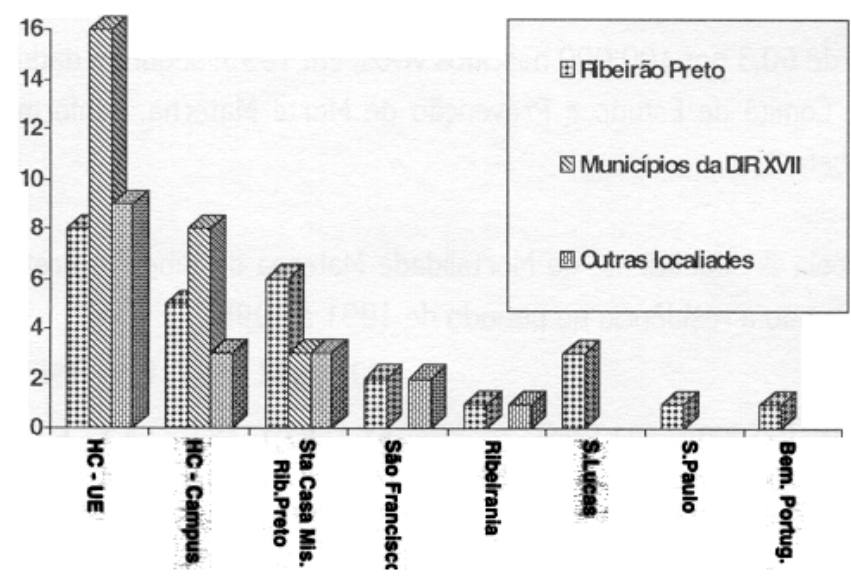

* Fonte: Secretaria Municipal da Saúde de Ribeirão Preto-SP e Comitê de Estudo e Prevenção de Morte Materna

Figura 2 - Morte Materna ocorrida em Ribeirão Preto, de acordo com o hospital e localidade no período de 1991 a 1995*

Observou-se que $44,6 \%$ das declarações de óbitos analisadas não dispõem de dados referentes ao momento em que ocorreu a morte materna. Entretanto, nas declarações em que há o preenchimento destes dados, observa-se que $52 \%$ dos óbitos maternos/residentes em Ribeirão Preto ocorrem no momento do parto, conforme Figura 3.

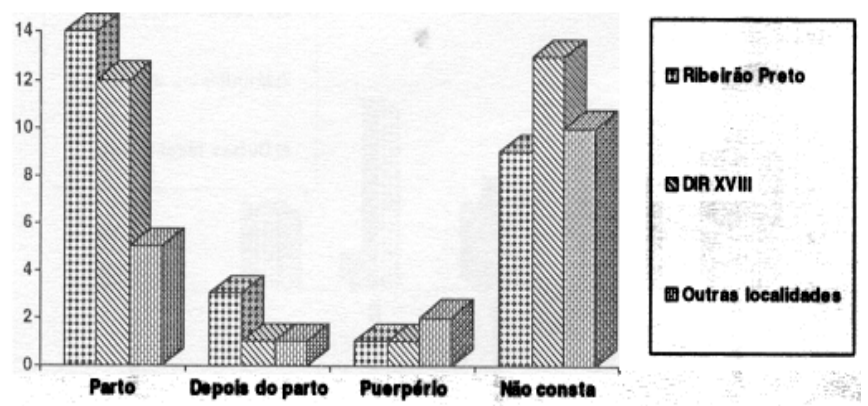

* Fonte: Secretaria Municipal da Saúde de Ribeirão Preto-SP e Comitê de Estudo e Prevenção de Morte Materna

Figura 3 - Morte Materna ocorrida em Ribeirão Preto, de acordo com a localidade e situação no período de 1991 a 1995*
Com relação ao tipo de parto, a investigação realizada na Maternidade Dona Evangelina Rosa ${ }^{(8)}$, aponta que a operação cesariana foi realizada em $60 \%$ das pacientes e constituiu-se na forma predominante de resolução da gravidez. Dados da literatura têm demonstrado que a operação cesariana constitui-se em importante fator de risco para óbitos maternos.

Das declarações analisadas do município Ribeirão Preto, observa-se que em $65 \%$ delas não constam informação referentes ao tipo de parto. Entretanto, nas declarações completas, constatase que $34 \%$ dos partos foram cesariana, conforme Figura 4.

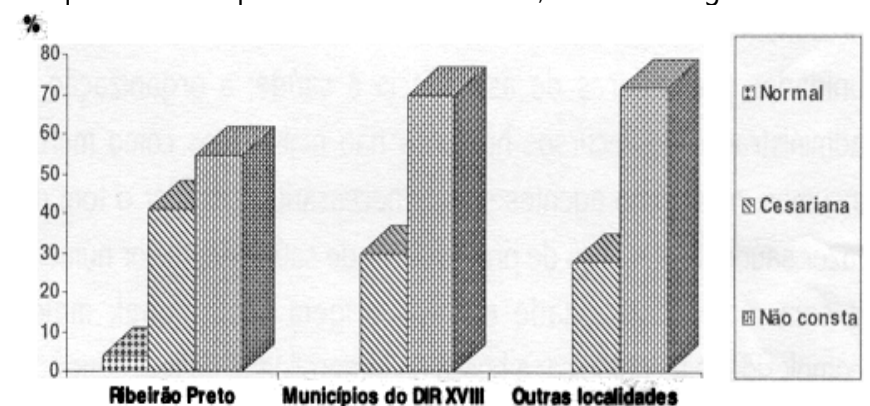

* Fonte: Secretaria Municipal da Saúde de Ribeirão Preto-SP e Comitê de Estudo e Prevenção de Morte Materna

Figura 4 - Morte Materna ocorrida em Ribeirão Preto, de acordo com o tipo de parto e localidade no período de 1991 a 1995*

Em pesquisa realizada no Hospital de Clínicas de Porto Alegre observa-se que, dos óbitos maternos registrados, 54,4\% foram em pacientes submetidas à cesariana. Tiveram como causa diretamente relacionada ao ato operatório/anestésico (48\%), infecção pós-cesariana (35\%), acidente anestésico (10\%), hemorragia $(7 \%)$. Verifica-se um risco relativo de morte associado ao procedimento cirúrgico é 28 vezes maior, em pacientes submetidas à cesariana ${ }^{(1)}$.

Em Ribeirão Preto, devido à baixa qualidade no preenchimento da declaração do óbito materno, não foi possível se constar a duração da gestação, pois em apenas quatro $(5,6 \%)$ das setenta e duas declarações analisadas constavam informação sobre a duração da gestação; estas, apresentaram uma variação de 28 a 37 semanas.

A investigação realizada na Maternidade Dona Evangelina Rosa ${ }^{(8)}$ mostra que a grande maioria dos óbitos maternos ocorreram na segunda metade da gestação $(96,43 \%)$, sendo que quase $40 \%$ do total, nesta pesquisa, ocorreu em mulheres com idade gestacional superior a 37 semanas.

Alguns avanços podem ser apontados em relação à assistência pré-natal em Ribeirão Preto: a proximidade da Unidade Básica Distrital de Saúde, ampliação da estrutura física e de recursos humanos, aquisição de aparelho de ultra-sonografia e cardiotocografia, a implantação da carteira de gestantes e da central de vagas para a área da obstetrícia ${ }^{(14)}$.

Estes avanços, foram possíveis, sem dúvida, graças ao processo de descentralização desencadeado nos últimos dez anos, "em várias áreas da administração pública brasileira e na saúde, 
principalmente a partir do Plano CONASP (Conselho de Saúde Previdenciária) e da estratégia das Ações Integradas de Saúde-AIS, elevando o município, ao principal papel de coordenador do processo de integração interinstitucional e posteriormente de responsável pela gestão de serviços públicos que atendessem às necessidades de saúde de seus munícipes. De certa forma, este processo traz à tona uma problemática fundamental que diz respeito à forma como os serviços de saúde do município são organizados e ofertados à população"(7).

Além das questões assinaladas acima, "é neste processo de (trans)formação que uma nova organização tecnológica do processo de trabalho em saúde se conforma e onde se fazem presentes: a necessidade de maior autonomia e poder decisório nas unidades prestadoras de assistência à saúde; a organização e administração de recursos humanos não mais vistos como meros insumos, mas como agentes sociais necessários a mudar o tom do fazer saúde; a presença de profissionais de saúde em maior número e com maior diversidade em sua origem profissional; maior complexidade tecnológica; a busca da integralidade da assistência; a presença da população nas decisões da saúde, com a constituição das Comissões Locais de Saúde"(7).

Apesar destes avanços, não foi possível nestes anos estabelecer uma referência hospitalar e/ou a contra-referência nos diversos níveis deste sistema de saúde. Observa-se a falta de integração entre o período pré-natal, parto e puerpério ${ }^{(15)}$.

$\mathrm{Na}$ investigação realizada no serviço de saúde municipal de Ribeirão Preto ${ }^{(16)}$, constata-se um perfil de mulheres que utilizam estas Unidades Básicas Distritais de Saúde de baixa escolaridade (71\% com primário incompleto), renda familiar de dois a quatro vezes o salário mínimo em $64 \%$ e nenhum tipo de convênio médico. Portanto, elas dependem única e exclusivamente do Sistema Único de Saúde para o acompanhamento pré-natal, resolução do seu parto e seguimento no puerpério.

Dessa forma, essas mulheres estarão mais propensas à morbi-mortalidade materna, uma vez que esta é um reflexo e sofre influência de fatores sócio-econômicos como a escolaridade e a renda familiar, somados à falta de referência hospitalar para resolução do parto.

Nos países desenvolvidos, onde a população apresenta padrão sócio-econômico satisfatório, com melhor estruturação dos sistemas de saúde e, conseqüentemente, de melhor assistência prénatal às gestantes, os índices de mortalidade materna mostram-se sensivelmente inferiores aos verificados em países subdesenvolvidos ${ }^{(8)}$.

Apesar dos avanços do Programa de Assistência Integral à Saúde da Mulher, observa no Brasil um coeficiente de mortalidade materna de 141 por 100000 nascidos vivos em 1990, que poderiam ser evitadas. As causas de óbito foram: toxemias (33\%), hemorragias $(21 \%)$, infecções $(17 \%)$, outras causas $(17,3 \%)$ e aborto $(9,7 \%)^{(17)}$.

No Canadá este coeficiente é de 4 por 100000 nascidos vivos $^{(17)}$. De acordo com o artigo "A absurda mortalidade materna", publicado em Medicina Conselho Federal ${ }^{(11)}$ o coeficiente de mortalidade materna na Argentina é de 50 por 100000 nascidos vivos, nos EUA 10 por 100000 nascidos vivos, na Holanda 6 por 100.000 nascidos vivos, em Cuba 7 por 100.000 nascidos vivos; no Brasil o índice está em 150 por 100.000 nascidos vivos, enquanto que no Canadá, o coeficiente é de 3 por 100000 nascidos vivos.

No Estado de São Paulo o coeficiente de mortalidade materna é de 53,7 por 100000 nascidos vivos, em 1990 e em 1995 é de 46,3, segundo a Fundação Sistema de Análise de Dados do Estado de São Paulo(SEADE).

Nas regiões mais pobres do Estado de São Paulo, como Botucatu, o índice de mortalidade materna está em torno de 180 por 100000 nascidos vivos ${ }^{(2)}$.

Em Ribeirão Preto, este coeficiente de mortalidade materna foi de 60,3 por 100000 nascidos vivos, em 1995, segundo dados do Comitê de Estudo e Prevenção de Morte Materna, conforme Tabela 2.

Tabela 2 - Coeficiente de Mortalidade Materna de Ribeirão Preto, segundo a residência no período de 1991 a 1995*

19911992199319941995

C.MM/100,000/res.Rib.Preto $\quad 51,4 \quad 87,1 \quad 48,6 \quad 84,4 \quad 60,3$ [MM/100.00/reside fora de Rib.Preto $141,5 \quad 87,1 \quad 60,9 \quad 96,4 \quad 156,8$ Total $\left[M M / 100,000 \mathrm{~N}, V^{*} \quad 192,9174,2109,5180,8217,1\right.$

** Fonte: Ribeirão Preto, Secretaria Municipal da Saúde, Plano de Saúde de Ribeirão Preto-SP; $1998^{(17)}$

A morte materna, nos países em desenvolvimento, é como uma epidemia silenciosa. É um problema de Saúde Pública não só pela sua magnitude, mas porque $90 \%$ das causas de mortes, durante o ciclo gravídico-puerperal, são evitáveis ${ }^{(2)}$. Em Ribeirão Preto também podemos observar este quadro, onde $68 \%$ destas mortes maternas são decorrentes de causas evitáveis, sendo as principais: hemorragias $(33,3 \%)$, outras causas $(26,4 \%)$, hipertensão (15,3\%), aborto (11,1\%), infeções $(8,3 \%)$ e puerpério (5,6\%), conforme Figura 5.

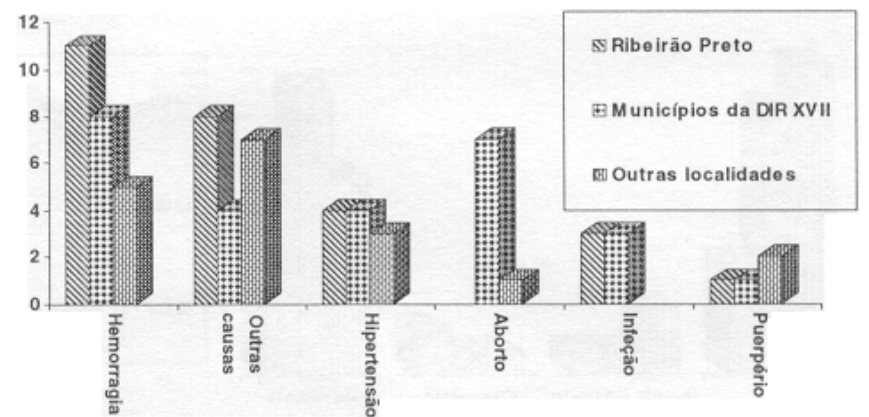

* Fonte: Secretaria Municipal da Saúde de Ribeirão Preto-SP e Comitê de Estudo e Prevenção de Morte Materna

Figura 5 - Mortalidade Materna ocorrida em Ribeirão Preto, de acordo com a causa e localidade no período de 1991 a 1995* 
Os dados encontrados são compatíveis com os obtidos na análise da mortalidade materna realizada no Centro Médico Universitário de Botucatu ${ }^{(2)}$ e do artigo "A absurda mortalidade materna" publicado em Medicina Conselho Federal ${ }^{(11)}$, ou seja, as principais causas de morte materna são as hemorragias, as infecções, a hipertensão, aborto, etc.

Na pesquisa realizada no Hospital de Clínicas de Porto Alegre consta que as principais causas de morte materna foram a hipertensão arterial (24\%), a infecção pós-cesárea (19\%), o aborto séptico $(9 \%)$, a AIDS $(3,5 \%)$ e outras causas $(53,5 \%)^{(1)}$.

Dados da Fundação Sistema de Análise de Dados do Estado de São Paulo (SEADE) em 1995, demonstram que 66,7\% dos óbitos maternos são de causas diretas, 22,2\% causas indiretas e 11,1\% abortos. Os principais grupos foram: hipertensão $(20,6 \%)$, parto normal e complicações que ocorrem durante o trabalho de parto e o parto $(18,7 \%)$, aborto $(11,1 \%)$, outras afecções existentes na mãe $(17,8 \%)$, outras categorias $(15,9 \%)$ e hemorragia $(6,0 \%)$ e complicações do puerpério $(9,8 \%)$.

0 predomínio das causas diretas reflete o padrão inadequado da assistência dispensada às gestantes e a necessidade da adoção de medidas urgentes que visem a melhoria na assistência e, por conseguinte, a prevenção da mortalidade materna ${ }^{(8)}$.

\section{CONSIDERAÇÕES FINAIS}

A pesquisa realizada no Hospital de Clínicas de Porto Alegre aponta para a necessidade de empreender-se um grande esforço na disseminação e melhoria da assistência pré-natal, principalmente

\section{REFERÊNCIA BIBLIOGRÁFICA}

1. Costa SM, Ramos JG, Cericatto R, Schilatter D, Petry MS. Causas de mortalidade materna no hospital de clínicas de Porto Alegre -RS análise de 15 anos. Rev Bras Ginecol Obstet 1997 agosto; 19(7):507-13.

2. Berezowski AT, Suetake H, Missiato M, Rudge MVC. Mortalidade materna análise dos últimos dez anos do Centro Médico-Universitário de Botucatu-UNESP. Rev Bras Ginecol Obstet 1995 novembro/ dezembro; 17(10):1001-795.

3. Nery IS. Perfil do atendimento à população feminina nos serviçoS públicos de saúde. Rev Bras Enfermagem 1996 outubro/dezembro; 49(4):497-510.

4. Oba MDV, Tavares MSG. 0 atendimento prestado às gestantes em unidades de saúde no município de Ribeirão Preto-SP: práticas de ações eventuais e curativas. Cogitare Enfermagem 1998 julho/ dezembro; 3(2):40-4.

5. Oba MDV, Tavares MSG. Aspectos positivos e negativos da assistência pré-natal no município de Ribeirão Preto-SP. Rev Latinoam Enfermagem 2000 abril; 8(2):11-7. dando-se ênfase na identificação precoce dos casos de doença hipertensiva específica da gestação e no estabelecimento de um sistema eficiente de referência e contra-referência entre os diversos níveis de complexidade do atual sistema de saúde ${ }^{(1)}$.

Com o trabalho desenvolvido por cada comitê regional, os índices coletados foram altíssimos, representando em algumas regiões mais de vinte vezes os valores observados em países desenvolvidos ${ }^{(8)}$.

Apesar dos dados obtidos terem sido comunicados aos órgãos competentes, como previamente propostos, assim como as causas no estabelecimento deste quadro e, em pelo menos um caso, terem sido sugeridas modificações pertinentes, não se tem notado significativos avanços que modifiquem os números relatados ${ }^{(2)}$.

É fundamental compreender que uma mulher não deve morrer por complicações devidas ou pioradas pela gravidez ou parto. A mortalidade materna é um problema de saúde pública que deve ser enfrentado, principalmente nos países subdesenvolvidos, com medidas de caráter geral, como a organização de pré-natal efetivo, o estabelecimento de sistema de referência hospitalar e amplas discussões sobre planejamento familiar ${ }^{(18)}$.

Compete as autoridades governamentais da área da saúde viabilizarem reestruturações dos serviços do Sistema Único de Saúde, com o objetivo de dar resolutividade a este desafio, demonstrando uma resposta efetiva aos problemas de saúde mais freqüentes na gestação, ou seja, proporcionando melhor qualidade na assistência à gravidez, ao parto e puerpério.

6. Oba MDV, Tavares MSG. Assistência pré-natal no município de Ribeirão Preto: sugestões e recomentações para ações futuras. Rev Paul Enfermagem 1997 janeiro/dezembro; 16(1/3):35-42.

7. Mishima SM. Constituição do gerenciamento local na rede básica de saúde em Ribeirão Preto [tese]. Ribeirão Preto (SP): Escola de Enfermagem de Ribeirão Preto/USP; 1995.

8. Valadares-Neto JD, Martins MPSS, Valadares SM, Costa-Filho NF. Mortalidade Materna na Maternidade Dona "Evangenila Rosa". Rev Bras Ginecol Obstet 1995 novembro/dezembro; 17(10):977-81.

9. Soares JWB, Soares JC, Taborda W, Bertini AM. Mortalidade materna. Femina 1997 agosto; 25(7):663-6.

10. Barbosa SA, Boyaciyan K, Marcus PAF, Veja CEP. Dificuldades encontradas para o estudo de casos de mortalidade materna na cidade de São Paulo (de 1993 a 1995). Rev Bras Ginecol Obstet 1997 abril; 19(3):179-83.

11. A absurda mortalidade materna. J Conselho Federal Medicina setembro/97; ano X, 85:18-9. 
12. Marcus PAF, Veja CEP, Boyaciyan K, Barbosa SA. Estudo da mortalidade materna no município de São Paulo durante o ano de 1995. Rev Bras Ginecol Obstet 1996 outubro; 18(9):731-6.

13. Sass N, Matar R, Camano L. Coeficiente de mortalidade materna geral e decorrentes de hipertensão arterial na Escola Paulista de Medicina da Universidade Federal de São Paulo no período de 1983

a 1993. Rev Bras Ginecol Obstet 1995 novembro/dezembro; 17(10):989-94.

14. Oba MDV. Assistência pré-natal: uma assistência integral ou fragmentada? [dissertação]. Ribeirão Preto (SP): Escola de Enfermagem de Ribeirão Preto/USP; 1996.

15 Oba MDV, Tavares MSG. As mulheres e os receios vivenciados em suas trajetórias obstétricas. Rev Bras Enfermagem 1996 outubro/ dezembro; 49(4):569-80.
16. Oba MDV, Tavares MSG. Quem são as mulheres e porque vão procurar o serviço de assistência pré-natal das unidades de saúde de Ribeirão Preto. Rev Bras Enfermagem 1999 outubro/dezembro; 52(4):596-605.

17. Ministério da Saúde (BR). Secretaria de Assistência à Saúde. Departamento de Programas de Saúde. Coordenação de Saúde Materno Infantil.Manual dos Comitês de Mortalidade Materna. Brasilia, 1994.

18. Oba MDV, Silva MG. Mortalidade Materna uma epidemia silenciosa no município de Ribeirão Preto-SP. Trabalho apresentado (apresentação oral) no $49^{\circ}$ Congresso Brasileiro de Enfermagem, Belo Horizonte (MG); 1997. 